\title{
КОНДЕНСАЦИОННЫЕ ТРОЙНЫЕ СОПОЛИМЕРНЫЕ ПОЛИАРИЛЕНЭФИРКЕТОНЫ: СИНТЕЗ И СВОЙСТВА
}

\author{
В.В. Шапошникова, С.Н. Салазкин
}

ФГБУН Институт элементоорганических соединений им. А.Н. Несмеянова РАН, Вавилова, ул 28, Москва, Российская Федерация, 119991

E-mail:vsh@ineos.ac.ru, snsal@ineos.ac.ru

В статье представлены данные о синтезе и свойствах аморфных статистических кардовых сополиариленэфиркетонов, получаемых поликонденсацией по механизму реакции нуклеофильного замещения активированного галогена в арилдигалогениде взаимодействием 4,4'-дифторбензофенона со смесью дикалиевых дифенолятов трех бисфенолов в N,N-диметилацетамиде. Синтез сополимеров с заданной молекулярной массой и характеризующихся большой структурной микронеоднородностью осуществлен при неэквимолярном соотношении мономеров (избытке бисфенолов) и блокировании концевых фенольных групп монофункциональным реагентом - 4-фторбензофеноном. Строение синтезированных сополимеров подтверждено данными ${ }^{13}$ С ЯМР, которые соответствуют предполагаемому строению со-ПАЭК. Показано, что синтезированные сополимеры обладают температурой начала размягчения от 180 до $240{ }^{\circ} \mathrm{C}$ и хорошими механическими свойствами.

Ключевые слова: термопласты, полиариленэфиркетоны, кардовые полимеры, статистические сополимеры, поликонденсация, реакция нуклеофильного замещения

\section{CONDENSATION TERNARY CO(POLYARYLENE ETHER KETONES): SYNTHESIS AND PROPERTIES}

\author{
V.V. Shaposhnikova, S.N. Salazkin
}

A.N. Nesmeyanov Institute of Organoelement Compounds, Russian Academy of Sciences, Vavilov, st. 28, Moscow, Russian Federation, 119991.

E-mail:vsh@ineos.ac.ru, snsal@ineos.ac.ru

The article presents data on the synthesis and properties of amorphous statistical cardo copolyarylene ether ketones obtained by polycondensation by the mechanism of nucleophilic substitution of activated halogen in aryldihalide by the interaction of $4,4^{\prime}$-difluorobenzophenone with a mixture of dipotassium diphenolates of three bisphenols in $N, N$-dimethylacetamide. The synthesis of copolymers with a given molecular weight and characterized by a large structural microheterogeneity was carried out at a non-equimolar ratio of monomers (excess of bisphenols) and blocking of the terminal phenolic groups with a monofunctional reagent - 4-fluorobenzophenone. The structure of the synthesized copolymers was confirmed by 13C NMR data, which correspond to the proposed structure of co-PAEK. It is shown that the synthesized copolymers have a softening onset temperature from 180 to $240^{\circ} \mathrm{C}$ and good mechanical properties.

Key words: thermoplastics, polyarylene ether ketones, cardo polymers, random copolymers, polycondensation, nucleophilic substitution reaction

Для цитирования:

Шапошникова В.В., Салазкин С.Н. Конденсационные тройные сополимерные полиариленэфиркетоны: синтез и свойства. Рос. хим. ж. (Ж. Рос. хим. об-ва). 2021. Т. LXV. № 3. С. 72-78

For citation:

Shaposhnikova V.V., Salazkin S.N. Condensation ternary co(polyarylene ether ketones): synthesis and properties. Ros. Khim. Zh. 2021. V. 65. N 3. P. 72-78 


\section{ВВЕДЕНИЕ}

Полиариленэфиркетоны относятся к классу термостойких термопластичных полимеров и благодаря комплексу ценных свойств (высокой термо-, тепло-, хемостойкости в сочетании с уникальными механическими, хорошими оптическими и электрофизическими свойствами) [1-6] находят широкое применение в технике [7], в том числе эксплуатируемой в экстремальных условиях. Синтез сополимерных ПАЭК, обладающих эффектом обратимой электронной проводимости в результате внешних воздействий (давления, температуры, магнитного поля и т.п.) [8, 9], открывает перспективы расширения областей применения этих полимеров, например, в электронике, фотонике, спинтронике.

Для получения статистических со-ПАЭК могут быть использованы различные подходы, а именно, взаимодействие 1) одного активированного дигалогенпроизводного с двумя и более бисфенолами [10-18], 2) двух и более активированных дигалогенпроизводных с одним бисфенолом [19], 3) двух и более активированных дигалогенпроизводных с двумя и более бисфенолами [20]. Увеличение структурной неоднородности статистических со-ПАЭК за счет сочетания в макромолекуле фрагментов различных дигалогенпроизводных и бисфенолов может способствовать появлению у сополимеров новых необычных свойств.

С целью получения сополимеров, характеризующихся большой структурной микронеоднородностью, в настоящей работе был осуществлен синтез аморфных статистических кардовых соПАЭК на основе 4,4'-дифторбензофенона и смеси трех бисфенолов и исследованы физико-механические свойства полученных сополимеров.

\section{ЭКСПЕРИМЕНТАЛЬНАЯ ЧАСТЬ}

Синтез аморфных статистических со-ПАЭК осуществляли поликонденсацией по механизму реакции нуклеофильного замещения активированного галогена в диарилгалогениде взаимодействием 4,4'-дифторбензофенона с дикалиевыми фенолятами смеси трех бисфенолов по методике, описанной в $[8,9]$ при неэквимолярном соотношении мономеров (избытке бисфенолов) и блокировании концевых фенольных групп монофункциональным реагентом - 4-фторбензофеноном.

Ниже в качестве примера приведена методика синтеза сополимерного ПАЭК на основе 4,4'дифторбензофенона и смеси бисфенолов: бисфенол А/ фенолфталеин/ фенолфлуорен (соотношение $0,25 / 0,5 / 0,25)$.
В предварительно продутую аргоном четырехгорлую колбу, снабженную мешалкой, термометром, трубкой для подачи аргона, насадкой для азеотропной отгонки воды, загружали 4,3204 г (0,0198 моль) 4,4'-дифторбензофенона, 1,1415 г (0,005 моль) бисфенола A, 3,1833 г (0,01 моль) фенолфталеина, 1,7500г (0,005 моль) фенолфлуорена, 0,0800 г (0,0004 моль) 4-фторбензофенона, 3,5935 г (0,026 моль) тщательно измельченного и прокаленного $\mathrm{K}_{2} \mathrm{CO}_{3}, 40$ мл N,N-диметилацетамида (ДМАА) и 20 мл хлорбензола. Колбу помещали в масляную баню, температуру в которой постепенно ( 0,5 ч) увеличивали до $185{ }^{\circ} \mathrm{C}$. Продолжительность синтеза после завершения отгонки азеотропной смеси хлорбензол - вода 7 ч. По окончании синтеза реакционную массу охлаждали и растворяли в хлороформе. Полученный раствор фильтровали от соли, промывали водой. Полимер выделяли в виде пленки путем упаривания его раствора в хлороформе при $25^{\circ} \mathrm{C}$ и сушили при постепенном повышении температуры от 60 до $140{ }^{\circ} \mathrm{C}$ в течение 18 ч, а затем при $160{ }^{\circ} \mathrm{C}$ в течение 25 ч.

Значения приведенной вязкости $\left(\eta_{п р}\right)$ определяли в хлороформе при $25{ }^{\circ} \mathrm{C}$ для растворов 0,05 г полимера в 10 мл растворителя на вискозиметре Уббелоде с диаметром капилляра 0,60 или 0,50 мм соответственно. Расчет $\eta_{п р}$ осуществляли по формуле:

$$
\eta_{\text {пр }}=\frac{\frac{\tau_{1}}{\tau_{0}}-1}{c},\left[\frac{\text { дл }}{\Gamma}\right]
$$

где $\tau_{0}$ - среднее значение времени истечения растворителя, сек; $\tau_{1}$ - среднее значение времени истечения раствора полимера, c; $c$ - концентрация раствора: 0,5 г полимера на 100 мл растворителя.

Спектры ${ }^{1} \mathrm{H}$ ЯМР и ${ }^{13} \mathrm{C}$ ЯМР для сополимеров регистрировали в лаборатории ядерного магнитного резонанса ИНЭОС РАН на спектрометре «Bruker» AMX-400 (400,13 и 100,61 МГц соответственно) для растворов в $\mathrm{CDCl}_{3}$, внутренний эталон $\mathrm{Me}_{4} \mathrm{Si}$. Отнесение сигналов в спектрах ${ }^{1} \mathrm{H}$ ЯMP и ${ }^{13} \mathrm{C}$ ЯМР осуществляли в соответствии с данными, рассчитанными по аддитивной схеме.

Пленки получали методом полива раствора 0,5 г сополимера в 10 мл хлороформа на целлофановую подложку.

Термомеханические испытания образцов сополимеров проводили на анализаторе TA Instruments TMA Q400 под нагрузкой 0,1 МПа со скоростью нагревания $1,5^{\circ} \mathrm{C} /$ мин в режиме сжатия. Измерения проводили в лаборатории полимерных матери- 
алов ИНЭОС РАН. За температуру начала размягчения ( $\left.\mathrm{T}_{\text {н.р. }}\right)$ полимера на термомеханической кривой принимали температуру, соответствующую точке пересечения касательных к двум ветвям кривой в области первого быстрого роста деформации.

Исследования механических свойств пленок осуществляли на динамометре типа «Поляни».
Измерения проводили в лаборатории полимерных материалов ИНЭОС РАН.

\section{РЕЗУЛЬТАТЫ И ИХ ОБСУЖДЕНИЕ}

Синтез аморфных статистических со-ПАЭК осуществляли по следующей схеме:

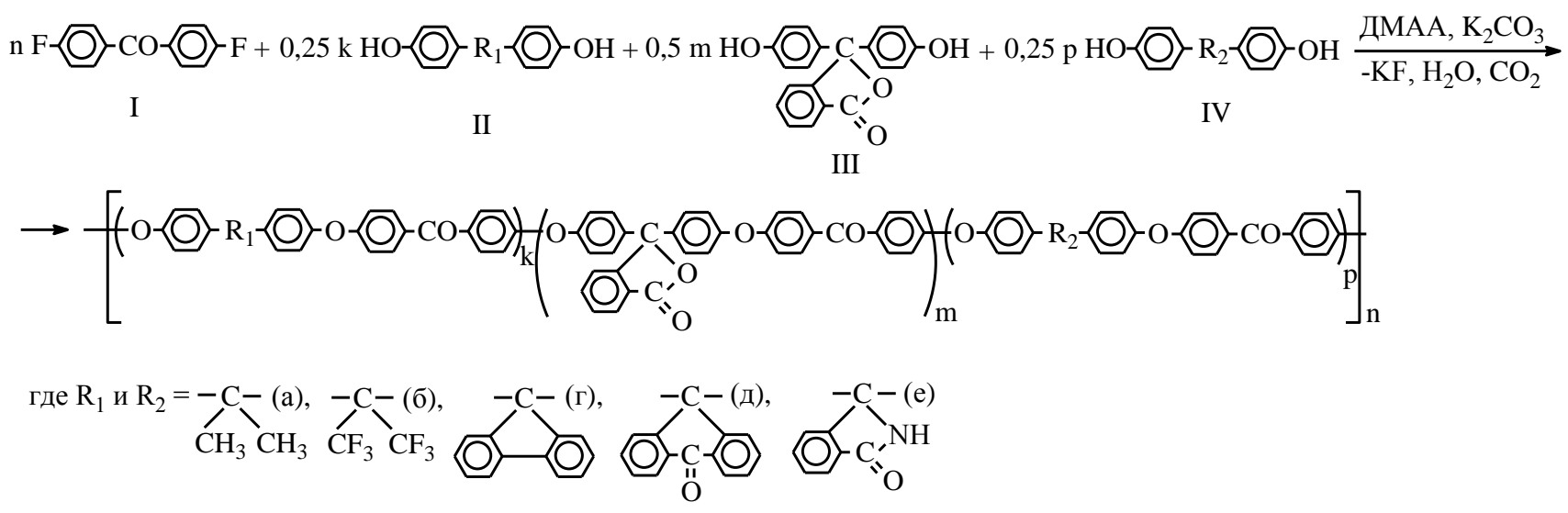

Попытка подтверждения строения синтезированных сополимеров данными спектроскопии ${ }^{1} \mathrm{H}$ ЯМР оказалась неудачной, т.к. на спектре ${ }^{1} \mathrm{H}$ ЯМР в области 6,5-8,5 ppm наблюдается множество перекрывающихся сигналов протонов, что обусловлено присутствием в макромолекулах сополимеров большого числа протонов. В связи с этим отнесение сигналов к конкретным протонам не представляется возможным.

Данные спектров ${ }^{13} \mathrm{C}$ ЯМР синтезированных сополимеров соответствуют предполагаемому строению со-ПАЭК. В качестве примера в табл. 1 представлены данные ${ }^{13} \mathrm{C}$ ЯМР для со-ПАЭК на основе соединения I и смеси бисфенолов: IIa, III и IVГ $(\mathrm{p} / \mathrm{q}=0,25 / 0,50 / 0,25)$. На спектре ${ }^{13} \mathrm{C}$ ЯМР этого сополимера пики $\mathrm{C}(1)$ и $\mathrm{C}(13), \mathrm{C}(49)$ и $\mathrm{C}(59), \mathrm{C}(29)$ и $\mathrm{C}(46), \mathrm{C}(16)$ и $\mathrm{C}(26), \mathrm{C}(4)$ и $\mathrm{C}(10), \mathrm{C}(52)$ и $\mathrm{C}(56)$, $\mathrm{C}(72)$ и $\mathrm{C}(78), \mathrm{C}(74)$ и $\mathrm{C}(80), \mathrm{C}(87)$ и $\mathrm{C}(97), \mathrm{C}(90)$ и C(94) наблюдаются в виде двух синглетных сигналов одинаковой интенсивности для каждой из пар перечисленных углеродов. Сигналы С(17), С(21), $\mathrm{C}(25), \mathrm{C}(27), \mathrm{C}(50), \mathrm{C}(54), \mathrm{C}(58), \mathrm{C}(60), \mathrm{C}(88)$, $\mathrm{C}(92), \mathrm{C}(96), \mathrm{C}(98)$ проявляются в виде двух сигналов одинаковой интенсивности для всех перечисленных углеродов. Сигналы С(32) и С(43) проявились в виде трех синглетных сигналов одинаковой интенсивности при $\delta=132,70 ; 132,85 ; 132,90$ м.д.. Это может быть обусловлено влиянием фталидной группы у атома C(35), которая не является симметричной по отношению к плоскости, в которой расположена основная цепь макромолекулы. Сигналы

$\mathrm{C}(22), \mathrm{C}(55)$ и $\mathrm{C}(93)$ также проявились в виде синглетов с одинаковой интенсивностью. Все выше перечисленные сигналы обусловлены статистическим распределением в макромолекуле фрагментов соединения I, имеющих различное окружение фрагментами бисфенолов.

Синтезированные со-ПАЭК растворимы в широком ряде органических растворителей (в хлороформе, метиленхлориде, ДМАА, тетрагидрофуране, диоксане и др.). Они образуют прозрачные прочные пленки. В таблице 2 представлены физико-механические характеристики соПАЭК, обладающих близкими значениями молекулярной массы, на основе соединения I и смеси трех бисфенолов.

Тройные со-ПАЭК характеризуются $\mathrm{T}_{\text {н.р. }}$ от 180 до $240{ }^{\circ} \mathrm{C}$ и хорошими механическими свойствами. Показатели прочности при разрыве пленок тройных и двойных со-ПАЭК сопоставимы, за исключением тройных сополимеров, содержащих фрагменты фенолантрона. В этом случае также отмечен необычно высокий (до 7,4 × $10^{3} \mathrm{MПа)} \mathrm{пока-}$ затель $\bar{E}$. Для выяснения причин такого изменения свойств пленок требуется дополнительное исследование. Высокие физико-механические показатели сополимерных ПАЭК позволяют рекомендовать их для создания материалов конструкционного назначения, а большая структурная микронеоднородность открывает перспективы получения на их основе электроактивных материалов. 
Таблица 1 Химические сдвиги и мультиплетности сигналов в спектре ${ }^{13}$ С ЯМР для сополимера на основе соединения I и смеси бисфенолов: IIa, III и IVг (p/q = 0,25/0,50/0,25), $\eta_{\text {пр }}=0,60$ дл/г

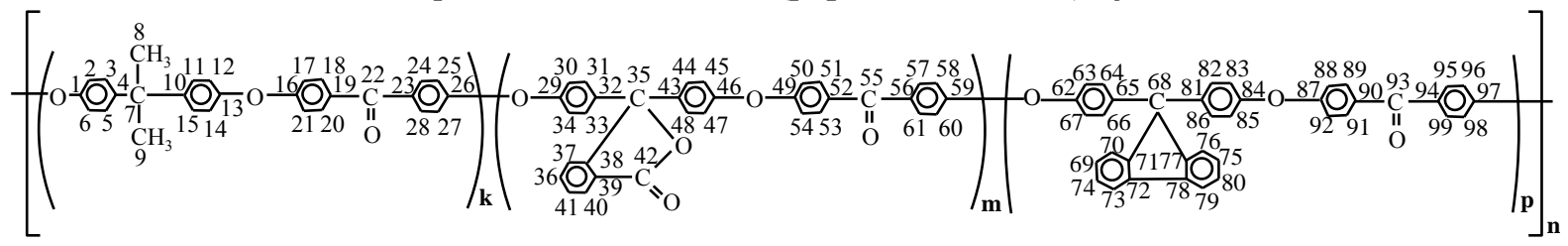

\begin{tabular}{|c|c|c|c|c|c|}
\hline Атом & $\delta_{\mathrm{c}}$, м.д. & Атом & $\delta_{c}$, м.Д. & Атом & $\delta_{\mathrm{c}}$, м.Д. \\
\hline $\mathrm{C}(1)$ & 161,$13 ; 161,25$ & $\mathrm{C}(29)$ & 156,$17 ; 156,23$ & $\mathrm{C}(62)$ & 161,$49 ; 161,60$ \\
\hline $\mathrm{C}(2)$ & 119,65 & $\mathrm{C}(30)$ & 117,83 & $\mathrm{C}(63)$ & 115,55 \\
\hline $\mathrm{C}(3)$ & 132,06 & $\mathrm{C}(31)$ & 129,04 & $\mathrm{C}(64)$ & 132,30 \\
\hline $\mathrm{C}(4)$ & 146,$78 ; 146,84$ & $\mathrm{C}(32)$ & 132,$70 ; 132,85 ; 132,90$ & $\mathrm{C}(65)$ & 140,06 \\
\hline $\mathrm{C}(5)$ & 132,06 & $\mathrm{C}(33)$ & 129,04 & $\mathrm{C}(66)$ & 132,30 \\
\hline $\mathrm{C}(6)$ & 119,65 & $\mathrm{C}(34)$ & 117,83 & $\mathrm{C}(67)$ & 115,55 \\
\hline$C(7)$ & 42,40 & $\mathrm{C}(35)$ & 91,01 & $\mathrm{C}(68)$ & 64,53 \\
\hline $\mathrm{C}(8)$ & 31,06 & $\mathrm{C}(36)$ & 134,51 & $\mathrm{C}(69)$ & 129,74 \\
\hline$C(9)$ & 31,06 & $\mathrm{C}(37)$ & 124,09 & $\mathrm{C}(70)$ & 124,09 \\
\hline $\mathrm{C}(10)$ & 146,$78 ; 146,84$ & $\mathrm{C}(38)$ & 151,82 & $\mathrm{C}(71)$ & 151,00 \\
\hline $\mathrm{C}(11)$ & 132,06 & $\mathrm{C}(39)$ & 125,50 & $\mathrm{C}(72)$ & 141,$94 ; 141,99$ \\
\hline $\mathrm{C}(12)$ & 119,65 & $\mathrm{C}(40)$ & 129,74 & $\mathrm{C}(73)$ & 120,44 \\
\hline $\mathrm{C}(13)$ & 161,$13 ; 161,25$ & $\mathrm{C}(41)$ & 126,$09 ; 126,29$ & $\mathrm{C}(74)$ & 127,$83 ; 127,96$ \\
\hline $\mathrm{C}(14)$ & 119,65 & $\mathrm{C}(42)$ & 169,53 & $\mathrm{C}(75)$ & 129,74 \\
\hline $\mathrm{C}(15)$ & 132,06 & $\mathrm{C}(43)$ & 132,$70 ; 132,85 ; 132,90$ & $\mathrm{C}(76)$ & 124,09 \\
\hline $\mathrm{C}(16)$ & 153,$40 ; 153,45$ & $\mathrm{C}(44)$ & 129,04 & $\mathrm{C}(77)$ & 151,00 \\
\hline $\mathrm{C}(17)$ & 117,$17 ; 117,38$ & $\mathrm{C}(45)$ & 117,83 & $\mathrm{C}(78)$ & 141,$94 ; 141,99$ \\
\hline $\mathrm{C}(18)$ & 128,41 & $\mathrm{C}(46)$ & 156,$17 ; 156,23$ & $\mathrm{C}(79)$ & 120,44 \\
\hline $\mathrm{C}(19)$ & 131,97 & $\mathrm{C}(47)$ & 117,83 & $\mathrm{C}(80)$ & 127,$83 ; 127,96$ \\
\hline $\mathrm{C}(20)$ & 128,41 & $\mathrm{C}(48)$ & 129,04 & $\mathrm{C}(81)$ & 140,06 \\
\hline $\mathrm{C}(21)$ & 117,$17 ; 117,38$ & $\mathrm{C}(49)$ & 160,$49 ; 160,62$ & $\mathrm{C}(82)$ & 132,30 \\
\hline $\mathrm{C}(22)$ & 194,20 & $\mathrm{C}(50)$ & 117,$17 ; 117,38$ & $\mathrm{C}(83)$ & 115,55 \\
\hline $\mathrm{C}(23)$ & 131,97 & $\mathrm{C}(51)$ & 128,41 & $\mathrm{C}(84)$ & 154,$44 ; 154,49$ \\
\hline $\mathrm{C}(24)$ & 128,41 & $\mathrm{C}(52)$ & 136,$62 ; 136,68$ & $\mathrm{C}(85)$ & 115,55 \\
\hline $\mathrm{C}(25)$ & 117,$17 ; 117,38$ & $\mathrm{C}(53)$ & 128,41 & $\mathrm{C}(86)$ & 132,30 \\
\hline $\mathrm{C}(26)$ & 153,$40 ; 153,45$ & $\mathrm{C}(54)$ & 117,$17 ; 117,38$ & $\mathrm{C}(87)$ & 160,$49 ; 160,62$ \\
\hline $\mathrm{C}(27)$ & 117,$17 ; 117,38$ & $\mathrm{C}(55)$ & 194,20 & $\mathrm{C}(88)$ & 117,$17 ; 117,38$ \\
\hline \multirow{11}{*}{$\mathrm{C}(28)$} & \multirow{11}{*}{128,41} & $\mathrm{C}(56)$ & 136,$62 ; 136,68$ & $\mathrm{C}(89)$ & 128,41 \\
\hline & & $\mathrm{C}(57)$ & 128,41 & $\mathrm{C}(90)$ & 136,$62 ; 136,68$ \\
\hline & & $\mathrm{C}(58)$ & 117,$17 ; 117,38$ & $\mathrm{C}(91)$ & 128,41 \\
\hline & & $\mathrm{C}(59)$ & 160,$49 ; 160,62$ & $\mathrm{C}(92)$ & 117,$17 ; 117,38$ \\
\hline & & $\mathrm{C}(60)$ & 117,$17 ; 117,38$ & $\mathrm{C}(93)$ & 194,20 \\
\hline & & \multirow{6}{*}{$\mathrm{C}(61)$} & \multirow{6}{*}{128,41} & $\mathrm{C}(94)$ & 136,$62 ; 136,68$ \\
\hline & & & & $\mathrm{C}(95)$ & 128,41 \\
\hline & & & & $\mathrm{C}(96)$ & 117,$17 ; 117,38$ \\
\hline & & & & $\mathrm{C}(97)$ & 160,$49 ; 160,62$ \\
\hline & & & & $\mathrm{C}(98)$ & 117,$17 ; 117,38$ \\
\hline & & & & $\mathrm{C}(99)$ & 128,41 \\
\hline
\end{tabular}


Таблица 2 Физико-механические свойства ${ }^{a}$ пленок аморфных статистических кардовых со-ПАЭК на основе I и смеси трех бисфенолов

\begin{tabular}{|c|c|c|c|c|c|c|c|c|}
\hline \multirow{3}{*}{ Бисфенолы } & \multirow{3}{*}{$\mathrm{k} / \mathrm{m} / \mathrm{p}$} & \multirow{3}{*}{$\begin{array}{l}\eta_{\text {пр }}, \\
\text { дл/Г }\end{array}$} & \multirow{3}{*}{$\underset{{ }^{\circ} \mathrm{C}}{\mathrm{T}_{\text {H.p. }},}$} & & & & & \multirow{3}{*}{$\begin{array}{c}\bar{E} \times 10^{-3}, \\
\text { МПа }\end{array}$} \\
\hline & & & & $\bar{\sigma}_{0}$ & $\sigma_{p}$ & $\bar{\varepsilon}_{0}$ & $\varepsilon_{p}$ & \\
\hline & & & & \multicolumn{2}{|c|}{ МПа } & \multicolumn{2}{|c|}{$\frac{1}{\%}$} & \\
\hline $\mathrm{IIa} / \mathrm{III} / \mathrm{IV} \Gamma$ & \multirow{10}{*}{$\begin{array}{l}0,25 / 0,5 / 0,25 \\
0,25 / 0,5 / 0,25\end{array}$} & 0,6 & 210 & 90 & 79 & 6 & 12 & 1,8 \\
\hline IIa/III/IVд & & 0,67 & 210 & 96 & 17 & 77 & 24 & 7,4 \\
\hline IIa/III/IVe & & 0,52 & 205 & 94 & 83 & 6,5 & 12 & 1,9 \\
\hline IIб/III/IVa & & 0,68 & 180 & 86 & 73 & 7 & 105 & 1,7 \\
\hline ІІб/III/IVд & & 0,72 & 215 & 89 & 74 & 7 & 59 & 2 \\
\hline IIб/III/IVГ & & 0,71 & 240 & 88 & 75 & 6 & 72 & 1,8 \\
\hline IIб/III/IVe & & 0,56 & 212 & 91 & 82 & 7 & 88 & 1,9 \\
\hline ІІг/III/IVe & & 0,56 & 240 & 95 & 82 & 7 & 17 & 1,9 \\
\hline ІІг/III/IVд & & 0,94 & 235 & 97 & 18 & 81 & 105 & 6,3 \\
\hline IIe/III/IVд & & 0,72 & 240 & 98 & 18 & 83 & 62 & 6,3 \\
\hline IIa & \multirow{6}{*}{$1 / 0 / 0^{\sigma}$} & 0,61 & 160 & 60 & 80 & 6,0 & 188 & 1,6 \\
\hline ІІб & & 0,53 & 170 & 68 & 60 & 5 & 58 & 1,6 \\
\hline III & & 0,70 & 230 & 90 & 95 & 6,2 & 190 & 2,0 \\
\hline ІІг & & 0,60 & 225 & 92 & 82 & 6,5 & 16 & 1,9 \\
\hline ІІд & & 0,58 & 240 & 90 & 83 & 7,6 & 8 & 1,8 \\
\hline IIe & & 0,54 & 260 & 107 & 97 & 9,0 & 10 & 2,0 \\
\hline $\mathrm{II} / \mathrm{III}$ & \multirow{3}{*}{$0,5 / 0,5 / 0^{\sigma}$} & 0,75 & 210 & 94 & 83 & 6,5 & 117 & 2,0 \\
\hline $\mathrm{II} / \mathrm{III}$ & & 0,72 & 230 & 88 & 75 & 8,0 & 35 & 1,6 \\
\hline $\mathrm{IIe} / \mathrm{III}$ & & 0,55 & 240 & $-{ }^{\mathrm{B}}$ & 110 & $-{ }^{\mathrm{B}}$ & 7 & 1,9 \\
\hline
\end{tabular}

${ }^{\text {а }} \bar{\sigma}_{O}$ - Предел вынужденной эластичности, $\bar{\sigma}_{p}$ - прочность в момент разрыва, рассчитанная на начальное сечение образца, $\bar{\varepsilon}_{0}$ - удлинение, соответствующее состоянию вынужденной эластичности; $\bar{\varepsilon}_{p}$ - относительное удлинение при разрыве, $\bar{E}$ - модуль высокоэластичности; бданные для гомо-ПАЭК и сополимеров на основе смеси двух бисфенолов приведены для сравнения; в пленки полимера разрушаются без образования шейки.

\section{ВЫВОДЫ}

В результате выполненного исследования установлена возможность получения структурно неоднородных аморфных кардовых тройных соПАЭК статистического строения, обладающих заданной молекулярной массой, на основе 4,4'-ди-

\section{ЛИ Т ЕР А Т У Р А}

1. Mullins M.J., Woo E.P. The Synthesis and Properties of Poly(Aromatic Ketones). J. Macromol. Sci. Part C. 1987. V. 27. N 2. P. 313-341. DOI: 10.1080/07366578708081918. фторбензофенона и смеси трех бисфенолов. Методом ${ }^{13} \mathrm{C}$ ЯМР подтверждено строение синтезированных сополимеров и изучены их физико-механические свойства. Показано, что со-ПАЭК обладают высокими физико-механическими свойствами.

Работа выполнена при поддержке Министерства науки и выстего образования Российской Федераичи.

\section{REFERENCES}

1. Mullins M.J., Woo E.P. The Synthesis and Properties of Poly(Aromatic Ketones). J. Macromol. Sci. Part C. 1987. V. 27. N 2. P. 313-341. DOI: 10.1080/07366578708081918. 
2. Rao V.L. Polyether ketones. J. Macromol. Sci. Part C. 1995. V. 35. N 4. P. 661-712. DOI: 10.1080/15321799508021753.

3. Kemmish D. Update on the technology and applications of polyaryletherketones. - Shawbury: Smithers Rapra Technology. 2010. $142 \mathrm{p}$.

4. Sabu T., Visakh P.M. Handbook of engineering and speciality thermoplastics: Volume 3: polyethers and polyesters. - Hoboken, New Jersey: John Wiley and Sons. Inc.; Salem, Massachusetts: Scrivener Publishing LLC. 2011. 562 p.

5. Шапошникова В.В., Салазкин С.Н. Исследования синтеза и свойств полиариленэфиркетонов. Известия Академии наук. Серия химическая. 2014. № 10. C. 2213-2223. DOI: 10.1007/s11172-014-0725-1.

6. Салазкин С.Н., Шапошникова В.В. Полиариленэфиркетоны - термо-, тепло- и хемостойкие термопласты и перспективы создания различных материалов на их основе. Высокомолекулярные соединения. Серия С. 2020. Т. 62. № 2. С. 108-121. DOI: $10.1134 / \mathrm{S} 1811238220020125$.

7. Михайлин Ю.А. Термоустойчивые полимеры и полимерные материалы. - СПб: Профессия. 2006. 624 с.

8. Пономарев А.Ф., Мочелев А.В., Ильясов В.Х., Лачинов А.Н., Салазкин С.Н., Шапошникова В.В., Шарапов Д.С., Корнилов В.M. Новые возможности эффективного влияния на транспорт зарядов во фталидсодержащих полиариленэфиркетонах. Высокомолекулярные соединения. Серия С. 2009. T. 51. № 7. C. 1300-1305. DOI: 10.1134/S1811238209010081.

9. Шапошникова В.В., Ткаченко А.С., Звукова Н.Д., Перегудов А.С., Клеменкова 3.С., Пономарев А.Ф., Ильясов В.Х., Лачинов А.Н., Салазкин С.Н. Новые возможности эффективного влияния на транспорт зарядов в полиариленэфиркетонах без использования фталидсодержащих фрагментов в полимерных цепях. Известия академии наук. Серия химическая. 2016. № 2. С. 502-506. DOI: 10.1007/s11172-0161329-8.

10. Zhang Y., Sun X., Niu Y., Xu R., Wang G., Jiang Z. Synthesis and characterization of novel poly(aryl ether ketone)s with metallophthalocyanine pendant unit from a new bisphenol containing dicyanophenyl side group. Polymer. 2006. V. 47. N 5. P. 1569-1574. DOI:10.1016/j.polymer.2005.12.068.

11. Yue X., Zhang H., Chen W., Wang Y., Zhang S., Wang G., Jiang $Z$. Crosslinkable fully aromatic poly(aryl ether ketone)s bearing macrocycle of aryl ether ketone. Polymer. 2007. V. 48. N 16. P. 4715-4722. DOI: 10.1016/j.polymer.2007.05.071.

12. Sun S., Guo M., Yi X., Zhang Z. Preparation and characterization of a naphthalene-modified poly(aryl ether ketone) and its phase separation morphology with bismaleimide resin. Polymer Bulletin. 2017. V. 74. P. 1519-1533. DOI: 10.1007/s00289-0161787-z.

13. Hou L., Wang Z., Xu J., Chen Zh. Poly(arylene ether ketone) containing amino and fluorenyl groups for highly selective of gas separation. Journal of Polymer Research. 2019. V. 26. N 243. DOI: 10.1007/s10965-019-1906-2.

14. Bao F., Zong L., Li N., Song Y., Pan Y., Wang J., Jian X. Synthesis of novel poly(phthalazinone fluorenyl ether ketone ketone)s with improved thermal stability and processability. Thermochimica Acta. 2020. V. 683. N 178184. DOI: 10.1016/j.tca.2018.12.028.
2. Rao V.L. Polyether ketones. J. Macromol. Sci. Part C. 1995. V. 35. N 4. P. 661-712. DOI: 10.1080/15321799508021753.

3. Kemmish D. Update on the technology and applications of polyaryletherketones. - Shawbury: Smithers Rapra Technology. 2010. 142 p.

4. Sabu T., Visakh P.M. Handbook of engineering and speciality thermoplastics: Volume 3: polyethers and polyesters. Hoboken, New Jersey: John Wiley and Sons. Inc.; Salem, Massachusetts: Scrivener Publishing LLC. 2011. 562 p.

5. Shaposhnikova V.V., Salazkin S.N. Synthesis and properties of poly(arylene ether ketones). Russian Chemical Bulletin. 2014. V. 63. N 10. P. 2213-2223. DOI: 10.1007/s11172-0140725-1.

6. Salazkin S.N., Shaposhnikova V.V. Poly(arylene ether ketones): Thermostable, Heat Resistant, and Chemostable Thermoplastics and Prospects for Designing Various Materials on Their Basis. Polymer Science. Series C. 2020. V. 62. N 2. P. 111-123. DOI: $10.1134 / \mathrm{S} 1811238220020125$.

7. Mikhailin Yu.A. Heat-resistant polymers and polymer materials. - SPb: Profession. 2006. 624 p.

8. Ponomarev A.F., Moshelev A.V., Il'yasov V.Kh., Lachinov A.N., Salazkin S.N., Shaposhnikova V.V., Sharapov D.S., Kornilov V.M. New possibilities for efficient effect of charge transport in phthalide-containing poly(arylene ether ketones). Polymer Science. Series C. 2009. V. 51. N 1. P.46-50. DOI: 10.1134/S1811238209010081.

9. Shaposhnikova V.V., Tkachenko A.S., Zvukova N.D., Peregudov A.S., Klemenkova Z.S., Ponomarev A.F., Il'yasov V.Kh., Lachinov A.N., Salazkin S.N. New possibilities for the effective influence on the charge transport in poly(arylene ether ketones) without using phthalide-containing fragments in the polymer chains. Russian Chemical Bulletin. 2016. V. 65. N 2. P. 502-509. DOI: 10.1007/s11172-016-1329-8.

10. Zhang Y., Sun X., Niu Y., Xu R., Wang G., Jiang Z. Synthesis and characterization of novel poly(aryl ether ketone)s with metallophthalocyanine pendant unit from a new bisphenol containing dicyanophenyl side group. Polymer. 2006. V. 47. N 5. P. 1569-1574. DOI: 10.1016/j.polymer.2005.12.068.

11. Yue X., Zhang H., Chen W., Wang Y., Zhang S., Wang G., Jiang Z. Crosslinkable fully aromatic poly(aryl ether ketone)s bearing macrocycle of aryl ether ketone. Polymer. 2007. V. 48. N 16. P. 4715-4722. DOI: 10.1016/j.polymer.2007.05.071.

12. Sun S., Guo M., Yi X., Zhang Z. Preparation and characterization of a naphthalene-modified poly(aryl ether ketone) and its phase separation morphology with bismaleimide resin. Polymer Bulletin. 2017. V. 74. P. 1519-1533. DOI: 10.1007/s00289-016-1787-z.

13. Hou L., Wang Z., Xu J., Chen Zh. Poly(arylene ether ketone) containing amino and fluorenyl groups for highly selective of gas separation. Journal of Polymer Research. 2019. V. 26. N 243. DOI: 10.1007/s10965-019-1906-2.

14. Bao F., Zong L., Li N., Song Y., Pan Y., Wang J., Jian X. Synthesis of novel poly(phthalazinone fluorenyl ether ketone ketone)s with improved thermal stability and processability. Thermochimica Acta. 2020. V. 683. N 178184. DOI: 10.1016/j.tca.2018.12.028. 
15. Zheng Y., Yang X., Yuan M., Luo J. Synthesis and gas transport properties of novel poly(ether ether ketone)s containing fluorene group. High Performance Polymers. 2019. V. 31. N 9-10. P. 1173-1182. DOI: 10.1177/0954008319832956.

16. Yang S., Ahn Y., Kim D. Poly(arylene ether ketone) proton exchange membranes grafted with long aliphatic pendant sulfonated groups for vanadium redox flow batteries. J. Mater. Chem. A. 2017. V. 5. P. 2261-2270. DOI: 10.1039/c6ta07456a.

17. Han X., Xie Y., Liu D., Chen Z., Zhang H., Pang J., Jiang Z. Synthesis and properties of novel poly(arylene ether)s with densely sulfonated units based on carbazole derivative. Journal ofMembrane Science. 2019. V. 589. N 117230. DOI: 10.1016/j.memsci.2019.117230.

18. Yang S., He Y., Liu Y., Leng J. Shape-memory poly(arylene ether ketone)s with tunable transition temperatures and their composite actuators capable of electric-triggered deformation. Journal of Materials Chemistry C. 2020. V. 8. N 1. P. 303-309. DOI: 10.1039/c9tc05475h.

19. Kawaguchi S., Morikawa A. Preparation of poly(ether ketone)s having phenyl, biphenyl, and terphenyl side groups and comparison of their properties. High Performance Polymers. 2018. V. 30. N 1. P. 67-75. DOI: 10.1177/0954008316679493.

20. Zhao Z., Gu Y., Chao D., Liu X. Synthesis and properties of shape memory poly(aryl ether ketone)s. European Polymer Journal. 2019. V. 116. P. 336-341. DOI: 10.1016/j.eurpolymj.2019.04.026.
15. Zheng Y., Yang X., Yuan M., Luo J. Synthesis and gas transport properties of novel poly(ether ether ketone)s containing fluorene group. High Performance Polymers. 2019. V. 31. N 9-10. P. 1173-1182. DOI: 10.1177/0954008319832956.

16. Yang S., Ahn Y., Kim D. Poly(arylene ether ketone) proton exchange membranes grafted with long aliphatic pendant sulfonated groups for vanadium redox flow batteries. J. Mater. Chem. A. 2017. V. 5. P. 2261-2270. DOI: $10.1039 / \mathrm{c} 6 \mathrm{ta} 07456 \mathrm{a}$.

17. Han X., Xie Y., Liu D., Chen Z., Zhang H., Pang J., Jiang Z. Synthesis and properties of novel poly(arylene ether)s with densely sulfonated units based on carbazole derivative. Journal ofMembrane Science. 2019. V. 589. N 117230. DOI: 10.1016/j.memsci.2019.117230.

18. Yang S., He Y., Liu Y., Leng J. Shape-memory poly(arylene ether ketone)s with tunable transition temperatures and their composite actuators capable of electric-triggered deformation. Journal of Materials Chemistry C. 2020. V. 8. N 1. P. 303-309. DOI: 10.1039/c9tc05475h.

19. Kawaguchi S., Morikawa A. Preparation of poly(ether ketone)s having phenyl, biphenyl, and terphenyl side groups and comparison of their properties. High Performance Polymers. 2018. V. 30. N 1.P. 67-75. DOI: 10.1177/0954008316679493.

20. Zhao Z., Gu Y., Chao D., Liu X. Synthesis and properties of shape memory poly(aryl ether ketone)s. European Polymer Journal. 2019. V. 116. P. 336-341. DOI: 10.1016/j.eurpolymj.2019.04.026.

Поступила в редакичию (Received) 01.08.2021 Принята к опубликованию (Accepted) 03.09.2021 